Gut, $1989, \mathbf{3 0}, 854-859$

Liver, biliary, and pancreas

\title{
Cytosolic and microsomal glutathione S-transferase isoenzymes in normal human liver and intestinal epithelium
}

\author{
P C HAYES, D J HARRISON, I A D BOUCHIER, L I MCLELLAN, \\ AND J D HAYES \\ From the University Department of Medicine, Royal Infirmary, Edinburgh, University Department of \\ Pathology, Edinburgh, and University Department of Clinical Chemistry, Royal Infirmary, Edinburgh.
}

SUMmARY Glutathione S-transferases are a group of drug metabolising and detoxification enzymes. We have studied the distribution of four isoenzymes, acidic, basic, neutral, and microsomal GST in human liver, gall bladder, and small and large intestinal epithelium by immunohistochemistry. Antibodies were raised in rabbits to purified GST subunits and several formalin fixed paraffin sections of each human tissue studied using the peroxidase-antiperoxidase method. Staining density was graded from very strong $(+++)$ to negative $(-)$. All four enzymes were identified within the liver, the acidic GST being found almost exclusively within the biliary epithelium. The gall bladder epithelium stained strongly for acidic and basic GST. In the small intestinal epithelium the acidic and neutral GST were readily identified in villi and crypts, whilst basic GST was found only in the villi and microsomal only in the crypts. In the colonic mucosa only acidic GST could consistently be identified. This histological heterogeneity may have functional implications for these enzymes in human hepatobiliary and intestinal tissue.

The glutathione S-transferases (GST) are a complex supergene family of detoxification enzymes found in a wide variety of animal tissues. These enzymes are multifunctional and their roles include the conjugation of lipophilic electrophiles with glutathione and the binding of a variety of non-substrate ligands. ${ }^{12}$ Substrates for GST include carcinogens and therapeutic drugs. The non-substrate ligands include compounds, such as bile acids, bilirubin and penicillin, that are bound non-covalently, as well as compounds such as 3-methylcholanthrene and dimethylaminoazobenzene, that are bound covalently. The former activity has led workers to suggest that hepatic GST may be involved with the intracellular carriage of lipophilic substances.

In man, the cytosolic GST isoenzymes are dimeric and can be subdivided, according to their isoelectric points into three major groups called 'acidic', 'basic', and 'neutral' GST. Each isoenzyme is dimeric, composed of $\mathrm{Yf}, \mathrm{Ya}$, or $\mathrm{Yb}$ subunits which have a molecular weight of 24800,26000 , and 26700

Address for correspondence: Dr P C Hayes. Dept of Medicine, Royal Infirmary, Fdinburgh $\mathrm{FH}_{3} \mathrm{gYW}$.

Accepted for publication 27 Octoher 1988 respectively. ${ }^{3} \mathrm{~A}$ fourth GST isoenzyme has recently been identified in human liver and has been purified from the microsomal subcellular fraction. By contrast with the cytosolic enzyme, this particulate enzyme contains a 17000 subunit, and is named microsomal GST. ${ }^{+5}$

Much has been learned about the biological control of GST. The molecular structure and the tissue specific expression of GST has been extensively studied in the rat." The histological localisation of GST, however, has received little attention. In the rat, the intrahepatic distribution of different GSTs has been studied ${ }^{7}$ whilst in man only the distribution of 'basic' GST has been described. ${ }^{* 4}$

The purpose of the present study was to identify the distribution of the four GST groups of enzymes within the normal liver, gall bladder and intestinal epithelium.

\section{Methods}

MATERIALS

Antisera to the cytosolic, basic, acidic, and neutral enzymes, as well as microsomal GST, were raised in 
Table Distribution of (GST isoenzymes in various tissues. The density of staining is indicated by: +++ very strong; ++ strong; + positive; \pm weak; - negative

\begin{tabular}{|c|c|c|c|c|c|c|}
\hline & \multicolumn{2}{|c|}{ Hepatocyte cytoplasm/nuclei } & Biliary epithelium & $\begin{array}{l}\text { Gall bladder } \\
\text { epithelium }\end{array}$ & $\begin{array}{l}\text { Small intestinal } \\
\text { epithelium }\end{array}$ & $\begin{array}{l}\text { Colonic } \\
\text { epithelium }\end{array}$ \\
\hline Acidic & \pm & - & +++ & ++ & ++ & ++ \\
\hline Basic & ++ & + & Larger ducts only & ++ & ++ (Not crypts) & - \\
\hline Neutral* & ++ & + & \pm & + & ++ & - or ++ \\
\hline Microsomal & + & - & + & \pm & - Crypts only & \pm \\
\hline
\end{tabular}

*The neutral GST is absent from about $50 \%$ of individuals.

rabbits according to the method described previously." "Briefly, $200 \mu \mathrm{g}$ of purified GST subunits was administered subcutaneously to rabbits on two occasions at six week intervals, initially in complete Freunds adjuvant and subsequently in incomplete Freunds adjuvant. The rabbits were then killed and serum collected. Specificity of the antisera was determined by Western blot analysis and radioimmunoassay.

Immunohistochemical staining was carried out using the peroxidase antiperoxidase (PAP) method of Sternberger:" as follows: formalin fixed paraffin sections of normal human liver tissue, gall bladder and intestinal epithelium of pathology archive material obtained from surgical specimens were treated with $1 \%(\mathrm{vol} / \mathrm{vol})$ hydrogen peroxide in methanol for 20 minutes to block the endogenous peroxidase activity. Sections were washed in Tris buffered saline (TBSpH 7.6) and treated with 20\% normal donkey sera (Scottish Antibody Production Unit, Carluke, UK) for 15 minutes before being incubated with the primary antibody $(1 / 20)$ for 30 minutes. Normal rabbit serum $(1 / 20)$ was used in place of the primary antibody for the negative controls. Incubations for 30 minutes with $1 / 30$ donkey antirabbit (Scottish Antibody Production Unit) and 1/100 rabbit peroxidase anti-peroxidase (Scottish Antibody Production Unit) were serially performed with TBS rinses and 10 minute incubations with $20 \%$ normal donkey sera between. Sections were subsequently treated with diaminobenzidine solution $(5 \mathrm{mg} / 10 \mathrm{ml}$ TBS $+0 \cdot 1 \quad \mathrm{M}$ imidazole $+100 \mu$ of 100 vol hydrogen peroxide) for 10 minutes. After thorough rinsing in tap water, sections were examined by light microscopy. Counterstaining was carried out with Meyer's haematoxylin.

\section{Results}

LIVER

All four GST isoenzymes were identified in human liver tissue although the pattern of staining differed (Table). Basic cytosolic GST was readily identified in both hepatocyte cytoplasm and nuclei (Fig. 1a) but only the larger biliary ductules showed positive staining. A negative control is illustrated in Figure $1 \mathrm{~b}$ for comparison.

Acidic cytosolic GST was weakly shown in hepatocyte cytoplasm, not in the nuclei but very strongly in

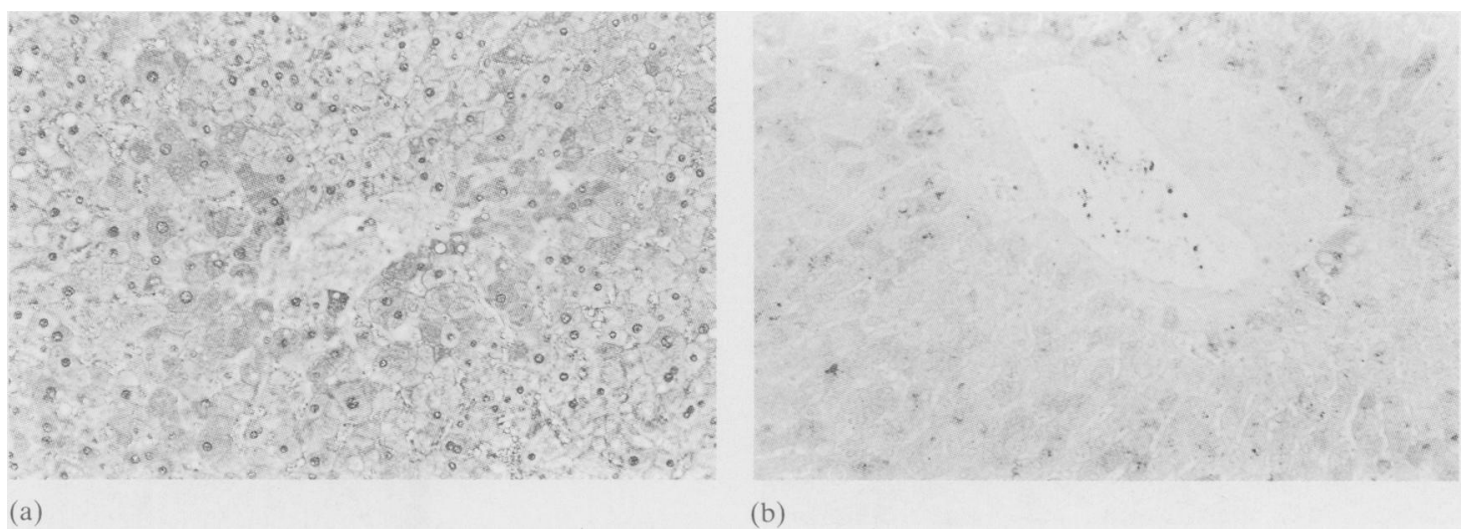

Fig. 1 (a) Distribution of basic GST in liver with dense staining in the cytoplasm of hepatocytes and their nuclei. (b) Negative control using non-immune rabbit antiserum. 


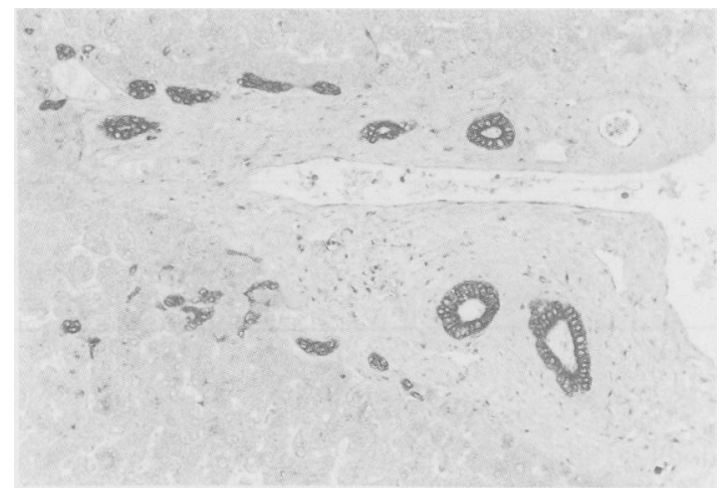

Fig. 2 Distribution of acidic GST in liver with weak staining in the cytoplasm of hepatocytes, negative nuclear staining and strong positive staining in the biliary epithelium.

the biliary ducts of all sizes (Fig. 2). The strongest staining was seen in the apical aspect of the cells of the biliary epithelium.

Neutral cytosolic GST was present in hepatocyte cytoplasm and nucleus (Fig. 3). Staining was primarily in Rappaport zone 1 (periportal) although weak positive staining was also observed in centrilobular hepatocytes. Biliary epithelium stained weakly.

Microsomal GST was seen in the cytoplasm of hepatocytes in a granular fashion (Fig. 4). The biliary epithelium was moderately stained.

\section{(i AI.I. BI.ADI)ER}

Strongly positive staining of the gall bladder epithelium was shown for acidic GST (Fig. 5) although weak positive staining for the basic and neutral isoenzyme was demonstrable.

SMALL INTESTINE

Acidic and neutral GST stained strongly (Figs 6 and

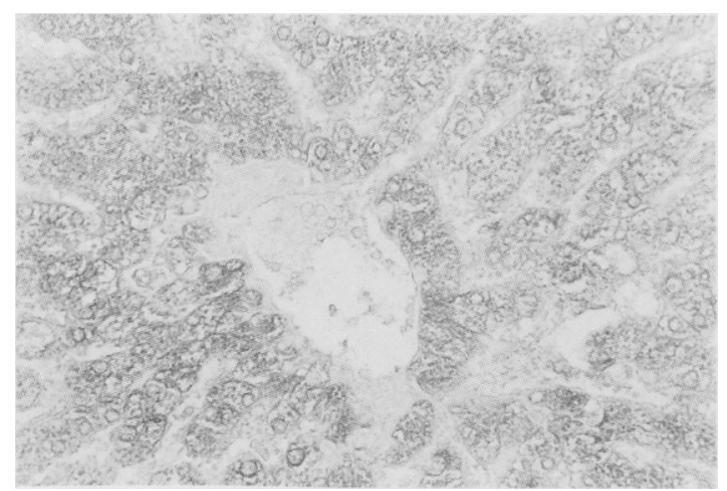

Fig. 4 Distribution of microsomal G.ST in liver with granular crtoplasmic and negative nuclear staining.

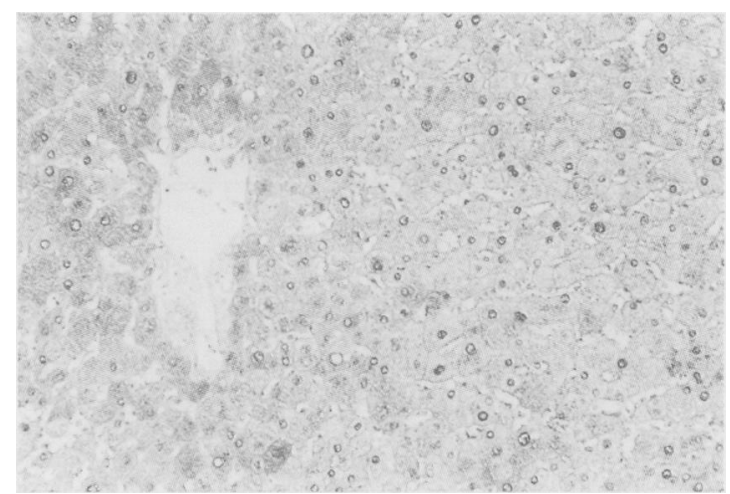

Fig. 3 Distribution of neutral GST in liver with moderate hepatocellular cytoplasmic staining and stronger nuclear staining.

7) in the intestinal cpithelium in the cells lining both the villi and crypts. Staining for basic GST was found in the cells lining the villi but not crypts (Fig. 8) whilst microsomal GST was found only in the cells lining the crypts (Fig. 9).

\section{L.ARGE INTESTINE:}

Only acidic GST could be consistently demonstrated easily in the colonic epithelium (Fig. 10) with basic and microsomal GST (Fig. 11). In some cases strong positive staining for neutral GST was found whilst others appeared negative.

\section{Discussion}

Glutathione S-transferases represent a group of isoenzymes which have important functions in the liver, including detoxification, drug metabolism and substrate carriage. Their existence in the human liver has been known for some time but their distribution within this organ and the biliary and intestinal

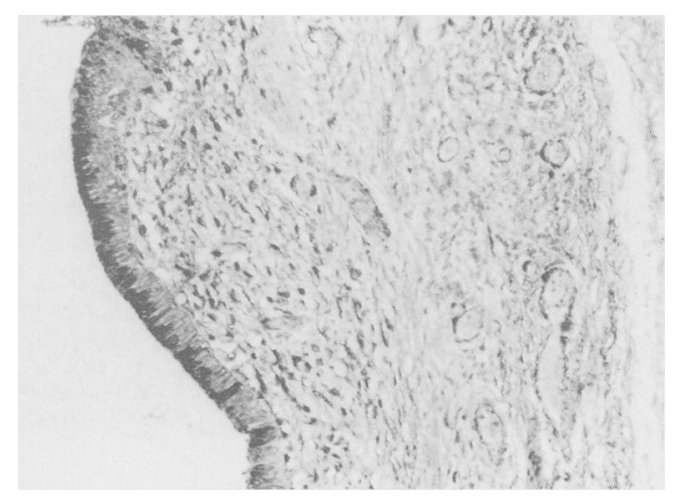

Fig. 5 Distribution of acidic (;ST in gall bladder with strong positive staining of the epithelium. 


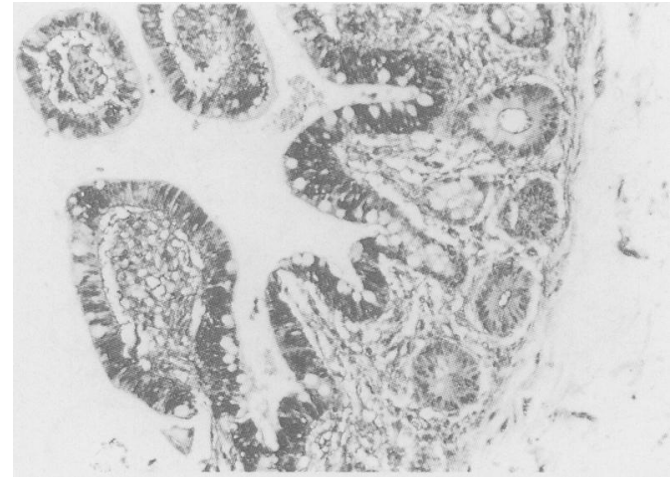

Fig. 6 Distribution of acidic GST in small intestinal epithelium with strong positive staining of epithelial villi and crypts.

epithelium is unknown and almost certainly has relevance for their biological function. In this study, we have shown that the four groups of glutathione $\mathrm{S}$-transferases can be readily identified in normal human liver tissue. The most striking difference in staining pattern was found for the acidic GST (YfYf) which occurred strongly and almost exclusively in the biliary epithelium. The staining identified small biliary radicals which otherwise could easily be overlooked. Such heterogeneity in distribution is not found in the rat. A difference in the hepatocyte nuclear staining was also apparent with the basic and neutral GST being present and microsomal and acidic GST absent. The reason for the nuclear localisation of basic and neutral GST is at present unclear. In the rat, the cytosolic GST Yb subunits bind DNA and Bennett et al' have reported an association between $\mathrm{Yb}$ GST polypeptides and nuclear chromatin. Indeed, these workers suggested that the $\mathrm{Yb}$ subunits are involved in nuclear RNA

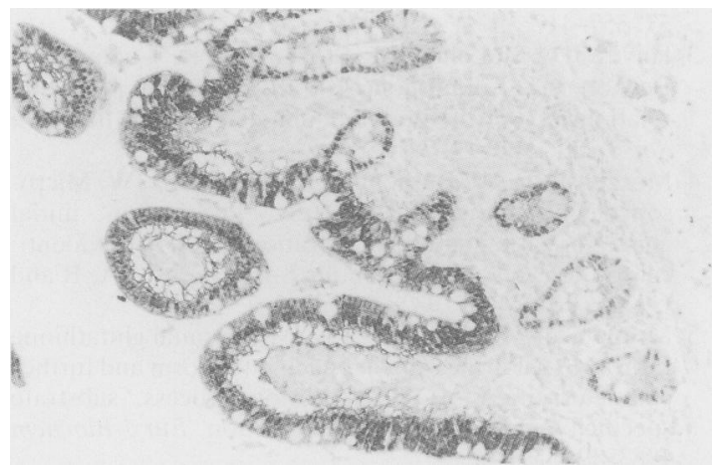

ig. 8 Distribution of basic GST in small intestinal epithelium with strong positive staining of the epithelial villi but not crypts.

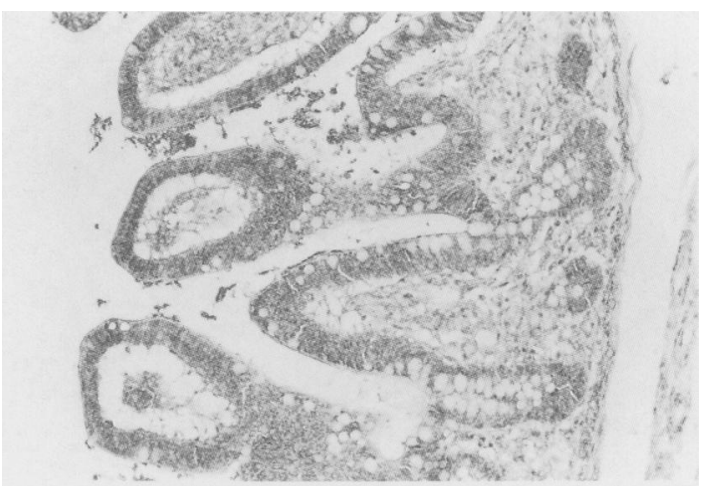

Fig. 7 Distribution of neutral GST in small intestinal epithelium with positive staining of both epithelial crypts and villi.

processing. Alternatively the human basic and/or neutral GST may serve in conjunction with DNA repair enzymes to repair peroxidised DNA since Ketterer $e t a l^{\text {:3 }}$ have shown that the rat GST isoenzymes exhibit peroxidase activity towards hydroperoxymethyl uracil as well as peroxidised DNA.

It has been shown using radioimmunoassays that basic cytosolic GST is a more sensitive marker of acute and chronic hepatocellular damage than aspartate or alanine aminotransferase $e^{1+}$ and may be increased in serum of patients with either alcoholic cirrhosis or autoimmune chronic active hepatitis in whom aspartate aminotransferase activity was normal. ${ }^{15}$ in $T$ The density of staining in the hepatocyte cytoplasm and nucleus shown in this study may explain this sensitivity. The acidic isoenzyme alone stained strongly in the biliary epithelium and may potentially be used as a marker for damage to this structure. The neutral GST is subject to polymorphism and in the Scottish population about $45 \%$ of

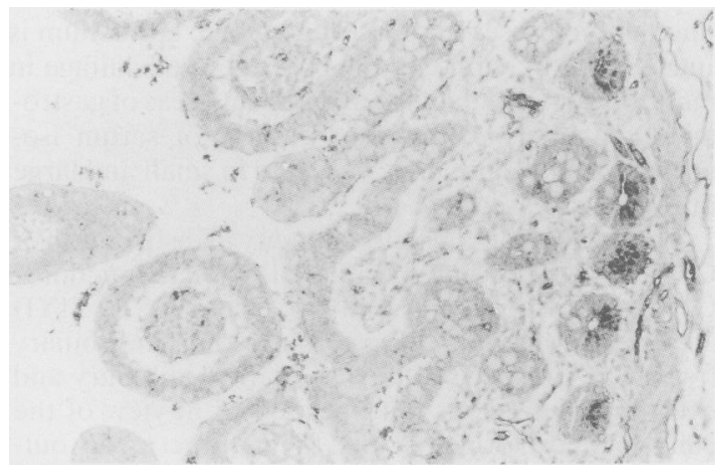

Fig. 9 Distribution of microsomal (;ST in small intestinal epithelium with negative staining of epithelial villi but positive staining of crypts. 


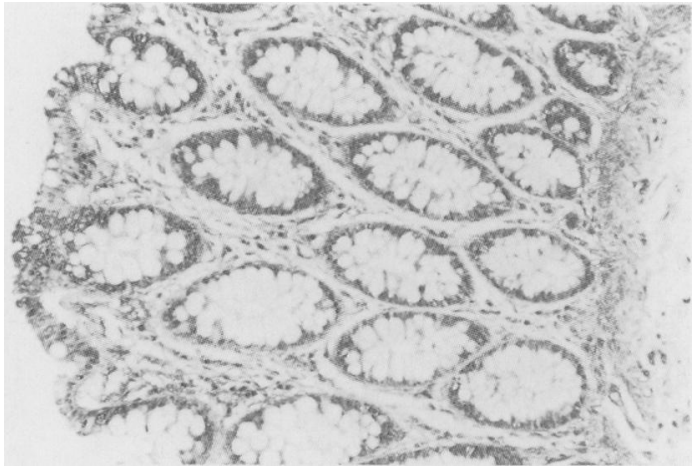

Fig. 10 Distribution of acidic GST in large intestinal epithelium with strong positive staining.

individuals fail to express $\mathrm{Yb}$ subunits. ${ }^{17}$ This enzyme cannot be used as a marker for tissue damage.

The heterogeneity of GST isoenzyme distribution was also evident in the extrahepatic tissues studied here. In the epithelium of the small intestine both the neutral and acidic GST was shown in the cells lining both the intestinal villi and crypts. The basic GST (YaYa) although present in the villi was absent in the crypt cells whilst the opposite situation was found for the microsomal isoenzyme. This suggests that as the cells mature the gene coding for the microsomal GST is switched off and the basic isoenzyme switched on. The biological significance of this is unclear but may have clinical relevance in the study of injured epithelium. In situations where cellular maturation is affected, differences in the tissue localisation of the two isoenzymes may be demonstrable.

In the colon only the acidic GST (YfYf) was consistently demonstrable in the epithelium. The presence of neutral GST (YbYb) was found in only some of the specimens of colon studied, an observation compatible with the known genetic variability in expression of this isoenzyme. The biological significance of the presence of GST in colonic epithelium is unclear but may potentially be used to advantage in monitoring tissue damage in different areas of gastrointestinal tract. A different pattern of serum isoenzyme abnormality may be found in small and large intestinal disease.

The reasons for the heterogeneity in distribution of GST isoenzymes may be related to their functions. Of the four isoenzymes, only the acidic GST (YfYf) appears throughout the gastrointestinal and biliary tract. The presence of this isoenzyme in biliary and extra biliary tissue is not unexpected in view of the embryological origin of the biliary trec as an outgrowth from the foregut. The function of acidic GST (YfYf) in these tissues is unclear but it may act as a carrier protein for biliary constituents such as bile

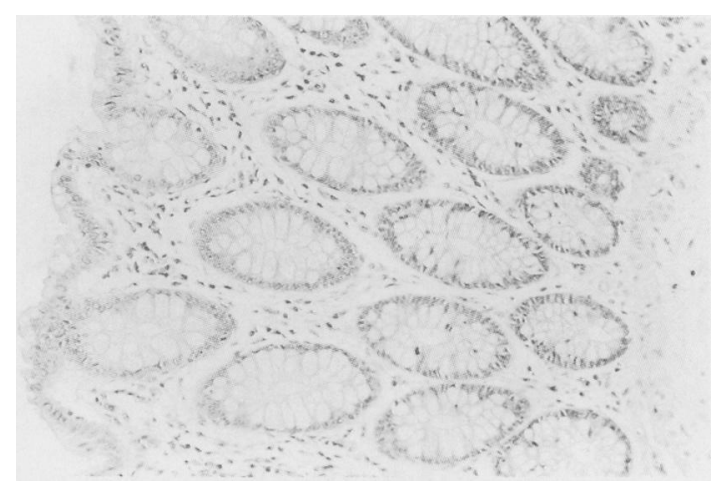

Fig. 11 Distribution of basic (;ST in large intestinal epithelium with negative staining.

acids, drugs and toxins; a function supported by the finding of large quantities of acidic GST in bile (unpublished observation).

In conclusion, we have shown that four different isoenzymes of GST can be identified in human liver, gall bladder and intestinal tissue. We believe that the heterogencity in distribution has functional implications and that further investigation of these enzymes in different disease states may have potential use diagnostically and in furthering our understanding of the basic biochemical changes taking place during disease progression.

We thank the MRC for financial support (G8713431) and Mrs M Rodriguez for typing the manuscript.

\section{References}

1 Jakoby WB. The glutathione S-transferases: a group of multifunctional detoxification proteins. Adv Enzymol Rel Areas Mol Biol 1977; 47: 383-414.

2 Mannervik B. The isoenzymes of glutathione S-transferases. Adv Enzymol Rel Areas Mol Biol 1985: 57: $357-417$.

3 Hayes JD, McLellan Ll. Stockman PK, Chalmers J, Beckett GJ. Glutathione S-transferases in man: the relationship between rat and human enzymes. Biochem Soc Trans 1987; 15: 721-5.

4 Morgenstern RF, Buthenberg C. De Pierre JW. Microsomal glutathione S-transferase. Purification, initial characterization and demonstration that it is not identical to the cytosolic glutathione S-transferases A. B and C. Eur J Biochem 1982; 1128: 243-7.

5 Morgenstern R, De Pierre JW. Microsomal glutathione transferase. Purification in unactivated form and further characterization of the activation process, substrate specificity and amino acid composition. Eur J Biochem 1983; 134: 591-7.

6 Hayes JD, Mantle TJ. Use of immuno-blot techniques to discriminate between the glutathione S-transferase $\mathrm{Yf}, \mathrm{Yk} \mathrm{Ya}, \mathrm{Yn} / \mathrm{Yb}$ and $\mathrm{Yc}$ subunits and to study their 
distribution in extrahepatic tissues. Evidence for three immunochemically distinct groups of transferase in the rat. Biochem J 1986; 23: 779-88.

7 Redick JA, Jakoby WB, Baron J. Immunohistochemical localisation of glutathione S-transferases in livers of untreated rats. J Biol Chem 1982; 257: 15200-3.

8 Campbell JAH. Bass NM, Kirsch RE. Immunohistological localization of ligands in human tissues. Cancer 1980; 45: 503-10.

9 Sherman M, Campbell JAH, Titmuss SA, Kew MC, Kirsch RE. Glutathione S-transferase in human hepatocellular carcinoma. Hepatology 1983; 3: 170-6.

10 Hayes JD, Gilligan D, Chapman BJ, Beckett GJ. Purification of human hepatic glutathione S-transferases and the development of a radioimmunoassay for their measurement in plasma. Clin Chim Acta 1983; 134: 10721.

11 Sternberger LA, Hardy PH, Cuculis JJ, Meyer HG. The unlabelled antibody enzyme method of immunohistochemistry: preparation and properties of soluble antigen-antibody complex (horseradish peroxidaseantihorseradish peroxidase) and its use in identification of spirochetes. J Histochem Cytochem 1970; 18: 315-33.

12 Bennett CF, Spector DL, Yeoman LC. Nonhistone protein $\mathrm{BA}$ is a glutathione $\mathrm{S}$-transferase localized to interchromatic regions of the cell nucleus. $J$ Cell Biol 1986; 102: 600-5.

13 Ketterer B, Tan KH, Meyer DJ, Coles B. Glutathione transferases: a possible role in the detoxification of DNA and lipid hydroperoxides. In: Mantle TJ, Pickett CB. Hayes JD, eds. Glutathione S-transferases and carcinogenesis. London: Taylor and Frances, 1987: 14963.

14 Beckett GJ, Hayes JD. Plasma glutathione Stransferase measurements and liver disease in man. J Clin Biochem Nutr 1987; 2: 1-24.

15 Beckett GJ, Hayes PC, Hussey AJ, Bouchier IAD, Hayes JD. Plasma glutathione S-transferase measurements in patients with alcoholic cirrhosis. Clin Chim Acta 1987; 169: 85-90.

16 Hayes PC, Hussey AJ, Keating J, et al. Giutathione S-transferase levels in autoimmune chronic active hepatitis: a more sensitive index of hepatocellular damage than aspartate transaminase. Clin Chim Acta 1988; 172: 211-6.

17 Hussey AJ, Hayes JD, Beckett GJ. The polymorphic expression of neutral glutathione $\mathrm{S}$-transferase in human mononuclear leucocytes as measured by specific radioimmunoassay. Biochem Pharmacol 1987; 36: 4013-5. 